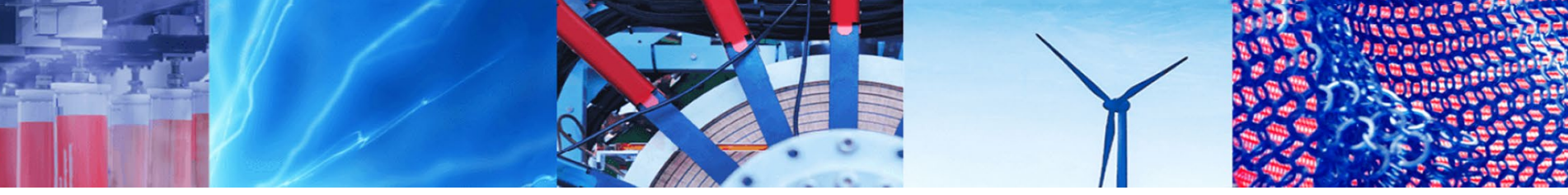

Review Paper

\title{
Membrane functionalization in artificial cell engineering
}

\author{
James W. Hindley ${ }^{1,2,3} \cdot$ Robert V. Law ${ }^{1,2,3} \cdot$ Oscar Ces $^{1,2,3}$
}

Received: 16 October 2019 / Accepted: 27 February 2020 / Published online: 10 March 2020

(c) The Author(s) $2020 \quad$ OPEN

\begin{abstract}
Bottom-up synthetic biology aims to construct mimics of cellular structure and behaviour known as artificial cells from a small number of molecular components. The development of this nascent field has coupled new insights in molecular biology with large translational potential for application in fields such as drug delivery and biosensing. Multiple approaches have been applied to create cell mimics, with many efforts focusing on phospholipid-based systems. This mini-review focuses on different approaches to incorporating molecular motifs as tools for lipid membrane functionalization in artificial cell construction. Such motifs range from synthetic chemical functional groups to components from extant biology that can be arranged in a 'plug-and-play' approach which is hard to replicate in living systems. Rationally designed artificial cells possess the promise of complex biomimetic behaviour from minimal, highly engineered chemical networks.
\end{abstract}

Keywords Artificial cell · Synthetic biology $\cdot$ Lipid vesicle $\cdot$ Responsive membrane $\cdot$ Compartmentalization

\section{Synthetic biology: top-down versus bottom-up?}

The past decade has seen accelerating progress towards an ambitious scientific goal - the development of a minimal cell. Two main approaches have been devised to achieve this: the top-down perspective aims to reduce the genetic composition of a single-celled organism until it contains the minimal number of genes necessary for cell survival $[1,2]$ whilst a second, alternative method has centred on the assembly of a minimal or artificial cell (AC) from its molecular building blocks. In such a bottom-up approach, reconstitution of aspects of cellular structure and function enables the study of specific processes decoupled from the complexity of biological systems, which to date has led to a better understanding of processes including membrane fusion [3] and fission [4]. Beyond generating fundamental understanding, the functions and behaviours of engineered ACs (e.g. chemical/biochemical production, triggered release, information processing) have the potential to be utilised in applications across biotechnology.

Whilst the approaches of top-down and bottom-up synthetic biology possess many differences, each lead to the fundamental question: what is a living system?

The chemoton model [5] proposed by Gánti defines that for a system to be 'alive', it needs three components; a boundary that creates a separate chemical space separate from its environment; an information system or template and a basic metabolism. Whilst this model encapsulates the key physical components of cellular life, it has been argued that this framework can be restrictive and lead to an overly narrow definition due to its focus on existing Earth-based biology [6]. One alternative currently adopted by NASA as a working definition of life proposes that 'life is a self-sustaining chemical system capable of Darwinian evolution' $[7,8]$. A third model proposed to adapt Turing's

$\triangle$ James W. Hindley, j.hindley14@imperial.ac.uk | ${ }^{1}$ Department of Chemistry, Imperial College London, Molecular Sciences Research Hub, Shepherd's Bush, London W12 OBZ, UK. ${ }^{2}$ Institute of Chemical Biology, Imperial College London, Molecular Sciences Research Hub, Shepherd's Bush, London W12 0BZ, UK. ' FABRICELL, Imperial College London, Molecular Sciences Research Hub, Shepherd's Bush, London W12 0BZ, UK. 
imitation game [9], defining the lifelike nature of an $A C$ by how successfully the $A C$ can mimic the behaviour of a living system [6]. Such a test has recently been implemented to test the efficiency of quorum sensing in a vesicle-based AC [10], indicating that such behavioural approaches may be especially useful until a universal definition of life has been reached.

Returning to the three-component chemoton model, the greatest amount of research to-date has focused on the structure and form of the boundary compartment. Due to the rapid development of different $\mathrm{AC}$ architectures and the central role of cell membranes in coordinating biological processes, this mini-review focuses on recent advances in lipid membrane construction/functionalization in bottom-up synthetic biology, and how these advances can be coupled with developments across biology, chemistry and nanotechnology.

\section{Constructing an artificial cell chassis}

A wide variety of materials have been used to create semi-permeable membranes, including phospholipids found in cell membranes $[11,12]$, synthetic polymerprotein conjugates that assemble at oil-water interfaces $[13,14]$, amphiphilic block co-polymers that form robust membranes [15-17], emulsion microdroplets stabilised via colloidal silica [18] and particles assembled layer-by-layer from oppositely charged polymers [19-21]. Membranefree systems have also been utilised, including liquid-liquid phase separated coacervates $[22,23]$ and the use of microfabrication methods to define the cell boundary [24-26]. Whilst microfabricated boundaries possess the advantage of high spatial control of content the molecular components of the AC cannot easily be decoupled from its fabricated chassis.

Although a multitude of approaches have been developed over the last decade, phospholipids and fatty acids still represent the most commonly used structural molecule for building ACs. This is unsurprising when we consider their central role in the development of the cell. Fatty acids are often used in protocell studies which aim to mimic the prebiotic conditions of the early Earth, and the development of the first cell membrane [27]. Whilst these systems will not be discussed here due to their low stability in complex media, interested readers are directed to a number of excellent recent reviews on the development of protocells $[27,28]$.

Long-chain phospholipids are biocompatible and selfassemble into varied structures at extremely low (nM) concentrations [29] driven by the hydrophobic effect [30] (Fig. 1a). Many diacylglycerol phospholipids have been used to construct lipid vesicles in aqueous solution

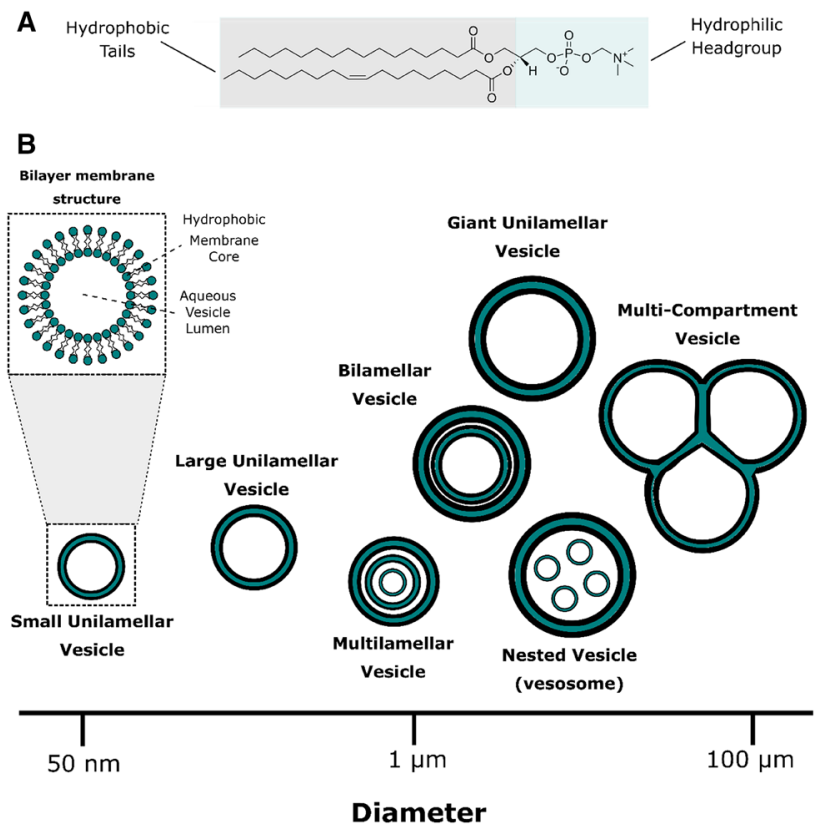

Fig. 1 Engineering vesicles across different length scales. a Structure of 1-palmitoyl-2-oleoyl-glycero-3-phosphocholine, a typical phospholipid. Lipid self-assembly into supramolecular structures is driven by the hydrophobic effect, minimising the interactions between hydrophobic tails and aqueous solution. b Vesicles can be generated from $\sim 50 \mathrm{~nm}$ to $\sim 50-100 \mu \mathrm{m}$. Traditional techniques such as sonication or extrusion can process hydrated multilamellar vesicles into large and small vesicles, whilst techniques such as electroformation and emulsion phase transfer can generate giant vesicles on the microscale. Smaller vesicles can be encapsulated inside to form nested vesicles, whilst bilamellar vesicles can be formed through sequential phase transfer processes. A crosssection of vesicular structure can be observed top left highlighting the bilayer structure wrapping around an aqueous lumen. Molecular content can be encapsulated within this lumen or within the hydrophobic core of the membrane depending on the physicochemical properties of the encapsulant

encapsulating an aqueous core. The increased stability of lipid vesicles compared to fatty acid vesicles makes them more suitable for use in applications such as drug delivery as well as structural elements of $A C s$, which often require complex media for the successful functioning of biological components. Lipid self-assembly has been studied for over 50 years [31,32], and from this work multiple production techniques have been refined that enable the user to create vesicles of controlled size and lamellarity (Fig. 1b). Nanoscale 'large' unilamellar vesicles (LUVs) can be assembled through thin-film hydration and extrusion [33] or solvent-based approaches [34] or hydrated vesicles can alternatively be processed below $100 \mathrm{~nm}$ through sonication to form 'small' unilamellar vesicles (SUVs) [35], whilst cell-size 'giant' unilamellar vesicles (GUVs) can be assembled via electroformation [36] or emulsion phasetransfer [37-39]. 
Electroformation uses the application of an alternating electric current to swell and bud off GUVs from a hydrated lipid film contained between two indium tin oxide (ITO) plates [36]. Whilst electroformation has been used extensively in biophysical studies to create vesicles with differing lateral phase behaviours [40-42], the mechanism of vesicle budding in electroformation prevents the encapsulation of large molecular-weight content, making it less suited for the development of lipid-based ACs as discussed below.

Emulsion-based methods are more promising for cell construction, offering users the ability to generate ACs with monolayer or bilayer compartment structure. Lipid(or surfactant-) stabilised water-in-oil (w/o) droplets generate containers where the monolayer interface acts as a barrier, isolating water-soluble cargo within the droplet. Droplet systems have been utilised to perform chemical and biochemical assays in high-throughput microfluidic formats [43]. If two monolayer-stabilised w/o droplets are brought together, the monolayers will combine upon contact to form droplet-interface bilayers (DIBs) (otherwise known as the contracting monolayer method) $[44,45]$. AC models can be created in droplets as well as in DIBs, using the lipid monolayer to demarcate the boundaries of the cell [46]. In DIB networks, each DIB acts as a semi-permeable membrane that allows the transport of molecular information between droplet compartments, opening the possibility for the construction of artificial tissues with controlled information pathways [47-49].

If lipid-stabilised microemulsions are transferred across a second w/o interface, GUVs are readily formed in a process known as emulsion phase-transfer (EPT) [37]. One major advantage of the EPT method compared to electroformation is its ability to form asymmetric lipid membranes more akin to biological systems [50]. This increases the biomimicry and functionality of the $A C$ by controlling the structure of each monolayer individually, enabling the production of vesicle bilayers with different physicochemical properties and molecular interactions on each face. One downside however is the use of oil in the production process. Whilst vesicles produced via EPT form stable membranes and can be used to reconstitute membrane pores such as the Staphylococcus aureus toxin alpha hemolysin (aHL) [51-53], the amount of oil included in such membranes varies $[37,54]$ and is yet to be quantified. This contrasts with electroformation where the aqueous production method ensures oil contamination of GUV membranes cannot occur.

The other primary advantage of EPT over electroformation is in its encapsulation behaviour. EPT is able to encapsulate water-soluble molecules size-independently with very high encapsulation efficiencies [37], whilst electroformation struggles to obtain such levels due to the mechanism of vesicle production. Bilayer swelling is reported to occur outwards from the multilamellar lipid sheets during electroformation [55], preventing the permeation of large and/or charged compounds. This advantage is extremely significant when building an $\mathrm{AC}$ as multiple components need to be encapsulated simultaneously with greatly differing molecular weights. This could include anything from small co-factors to ribosomes to plasmid DNA $(<1 \mathrm{kDa}, \sim 2,600 \mathrm{kDa}[56]$ and $\sim 3-60,000 \mathrm{kDa}$ [57] respectively). Successful reconstitution of cell-free expression has been carried out numerous times in GUVs generated via EPT which is testament to the high encapsulation efficiencies across a wide range of molecular weights $[11,58,59]$, boding well for the future production of ACs with increased complexity.

Smaller vesicles can also be utilised as components in an AC [60]. Enveloping vesicles within such a chassis creates nested vesicles (also known as vesosomes), which show promise in triggered release applications where the external lipid membrane acts as a boundary preventing degradation and content loss from the encapsulated vesicles within $[61,62]$. Such structures can be quickly generated via EPT and possess considerable potential as cell mimics with the encapsulated vesicles functioning as artificial organelles. One disadvantage to using nested vesicles as AC models lies in the poorly defined spatial organization of internal compartments. Encapsulated vesicles can also lack connectivity between compartments, however this can be overcome through membrane functionalization as discussed below.

Beyond its encapsulation abilities, EPT has also been used to create microscale ACs with varying numbers of compartments $[52,63]$. Such multi-compartment vesicles (MCV) can be created through the deposition of multiple lipid-stabilised w/o droplets that subsequently create GUVs linked by a single bilayer membrane. This approach yielded vesicles with multiple compartments, and an enzyme cascade was successively segregated within each internal space of a three-compartment vesicle [63]. Similar multi-compartment vesicles have been created from w/o/w double emulsions using microfluidics, where the vesicle size and compartment number can be tightly controlled [64]. This was achieved through the use of an additional surfactant, which modulates the interfacial energy and helps control the dewetting of MCVs from the oil phase. Using this approach MCVs of varying compartment number could be controllably produced (Fig. 2). Such microfluidic approaches reduce the difficulty in MCV assembly associated with manual liquid handling as well as increasing the throughput of vesicle production. 
Fig. 2 Forming multicompartment vesicles via microfluidics. Multi-compartment vesicles are controllably formed through the co-encapsulation of two different droplet species into w/o/w emulsions. Addition of Pluronic F- 68 to aqueous solution triggers de-wetting of the oil from the droplets, generating oil-free multi-compartment vesicles with controlled compartment number. Reprinted with permission from [64]. Copyright 2016 American Chemical Society

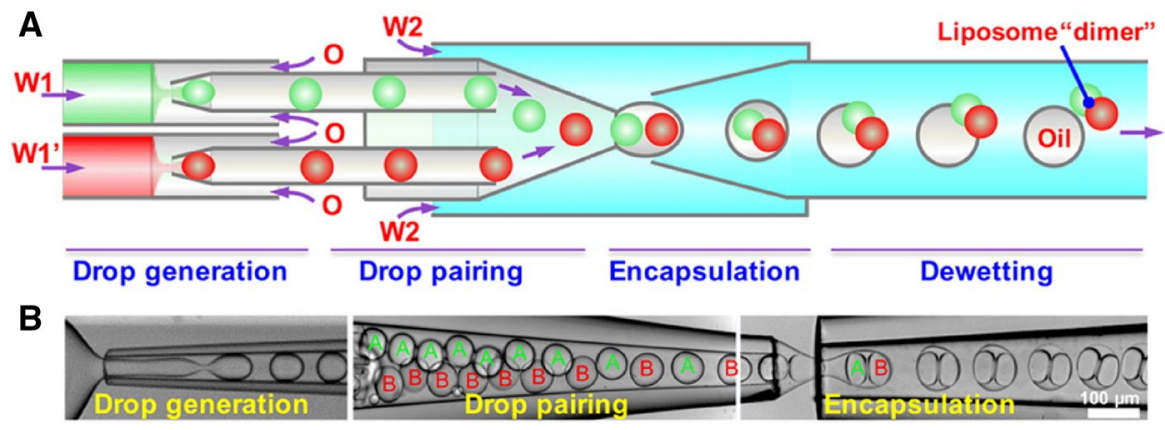

C
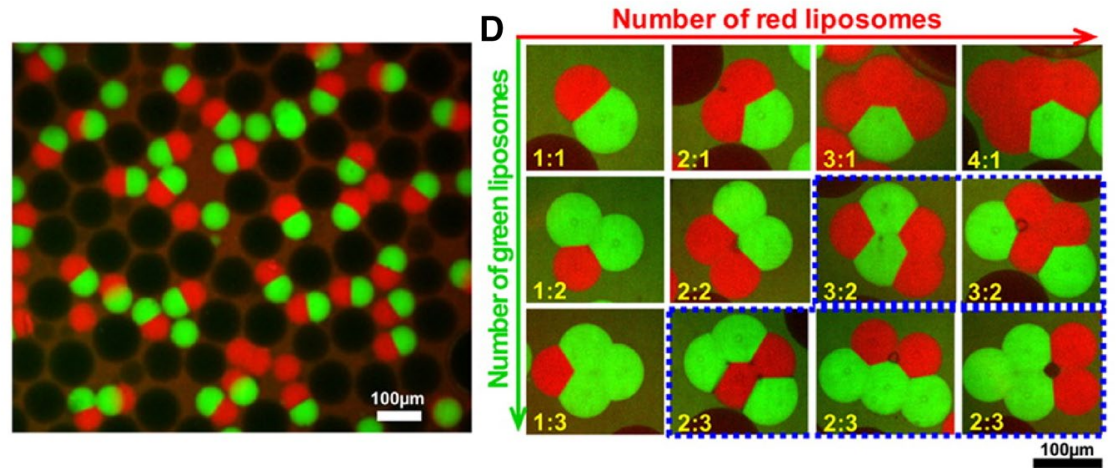

\section{Using molecular motifs to drive artificial cell function}

Advancements in membrane engineering have led to the creation of different molecular architectures that can be constructed and combined to form higher-order structures $[47,63,65,66]$. After these significant developments, new questions arise: how can the cell architecture be networked with the other components of the AC, and how can the cell chassis be incorporated as an active element in cell function?

Whether considering the creation of artificial cells or 'smart' soft materials for application, in each case the construct needs to become programmable. This can be achieved at a molecular level using chemical, physical or biological motifs, including the use of different chemical functional groups embedded in the cell mimic, functionalising the cell with the repertoire of biomolecules found in nature or alternatively, using the phase behaviour of lipid membranes as responsive elements in an artificial cell wall. Thinking more ambitiously, one could imagine engineering hybrid systems which use whole cells or organelles as components of a complex material [67-69].

Integrating molecular motifs into lipid membranes is a powerful way to generate artificial cell function (Fig. 3). Many efforts have focused on generating synthetic lipids that provide drug delivery formulations with a specific trigger that can respond to disease states in vivo, including $\mathrm{pH}$ $[70,71]$, enzymes [72-75], temperature $[76,77]$ or redox state [78]. These can be repurposed as elements in artificial cells, where instead of releasing a drug in response to a disease trigger, the membrane acts as a responsive part of a larger ensemble. Alternative motifs such as nanoparticles or polymerizable lipids can be included to generate vesicle-based systems that respond to exogeneous stimuli such as light $[79,80]$, ultrasound [81] or applied magnetic fields [82, 83].

In each case, the responsive element can act reversibly or irreversibly. Many nanomedical triggered-release systems are irreversible, designed to release their entire payload over time [84]. Examples of reversible systems are rarer, generally focusing on the response of the membrane to transient heating events. This includes gel-phase liposomes that have been shown to leak cargo as they undergo a phase transition from the gel to fluid states at a characteristic melting temperature $\left(\mathrm{T}_{\mathrm{m}}\right)$ [85], and more recently three-component (ternary) compositions were shown to possess content release properties dependent on the mixing temperature $\left(T_{\text {mix }}\right)$ of the formulation [86]. Leakage occurs at these temperatures due to incompatibilities in membrane packing and hydrophobic matching at forming/melting domain boundaries [87], temporarily increasing membrane permeability.

Once activated, some functional groups act by destroying the entire membrane [88] whilst other create pores in an otherwise stable membrane $[79,89,90]$, facilitating the size-selective passage of solutes across the membrane. When considering derivatisation of vesicles for building 


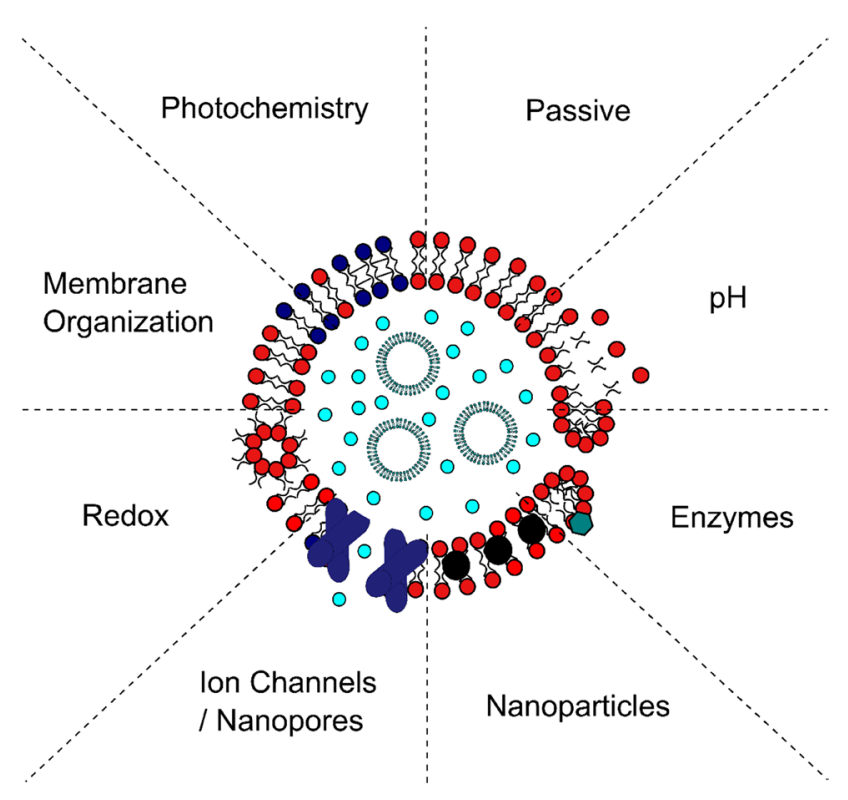

Fig. 3 Generating responsive artificial cells using molecular functionalization. A variety of molecular motifs can be used to generate stimulus responsive permeability changes in lipid ACs. This includes; tailoring the lipid composition to respond to enzymes such as phospholipases; tailoring the pKa of lipids to fashion $\mathrm{pH}-$ response; the inclusion of nanoparticles to generate optically- or magnetically-induced membrane fusion; ion channel or DNA nanopore reconstitution to generate chemical/mechanical-response; dithiol reduction to modulate membrane composition and hence permeability; lateral phase separation or gel-liquid melting transitions to generate membrane discontinuities at compositiondependent temperatures and photopolymerisation to trigger temporary membrane defects. Vesicle membranes are encapsulated alongside small molecules to illustrate that functional membranes can be incorporated to trigger intra-cellular events as well as content exchange across the external membrane of the $A C$

artificial cells, an ideal system would provide the transient generation of nanopores in a stable membrane. This enables intra- and inter-cellular communication through content mixing without destroying the benefits of compartmentalization within the artificial cell. This is particularly true when considering functionalisation of the external boundary of the cell, as membrane breakdown here will lead to loss of cell contents (and hence cell function).

One example of a nested vesicle that uses membrane biophysics to control reactor function was developed by Vogel and co-workers [90, 91]. In this work, triggered release of different enzyme substrates occurs from two populations of encapsulated vesicles that are activated at successively increasing $T_{m}$, triggering reactor function in a programmed fashion [91]. One disadvantage to using temperature for control of chemical reactions in ACs is the lack of orthogonality: in order for reaction 2 to activate, reaction 1 must also be activated. The same disadvantage is present for other endogenous stimuli such as $\mathrm{pH}$; such stimuli are generally best used to control singular processes in ACs. Alternatively, exogenous stimulus response (e.g. light) enables selective activation of different processes leading to the creation of vesicle modules capable of orthogonal function. Optical control over AC reaction processes has been recently demonstrated by various laboratories, from the use of diacetylene photopolymerisation for controlling enzyme catalysis in nested vesicles [92] to using photocaged RNA polymerase to control cell-free transcription-translation in a minimal tissue [48].

Purified components of biological systems represent another rich source for generating functional membranes [93-96]. Proteins represent an obvious candidate for vesicle functionalisation due to their ubiquitous presence across biology. In one example, synthetic chemistry was combined with enzyme catalysis by Devaraj and coworkers to generate vesicle in situ [97]. In this work, the soluble enzyme FadD10 was used to produce adenylated fatty acids that react with amine-functionalised lysolipids, generating membrane forming phospholipids. Such efforts highlight how synthetic chemistry can be used to reduce the number of biochemical steps necessary for function (1 catalytic step in the minimal system versus 3 in the Kennedy cycle [98]) whilst maintaining the same functional effect (in this case phospholipid production and membrane growth).

Another set of proteins studied in bottom-up systems is the Min system from Escherichia Coli. The system consists of MinC, D and E which together positions the bacterial cell division machinery at the centre of the cell [99]. The Schwille group has successfully reconstituted MinD and E into surface-attached lipid bilayers (SLBs) [100], microemulsion droplets [101] and giant vesicles [102] in order to study system function ex vivo. This enabled observation of oscillatory protein complex assembly at membrane interfaces, generating multiple oscillatory behaviours in giant vesicles including 'breathing,' 'circling' and sphere to dumbbell morphological changes based on vesicle size and encapsulated protein concentration [102]. The Min system represents an excellent example of how a single two-protein complex can dynamically affect membrane morphology, whilst simultaneously showing the utility of model membrane systems in understanding fundamental cell biology.

The assembly/disassembly of the Min complex is just one of many energy intensive processes essential for biological homeostasis. The generation of energy in artificial cells is therefore essential for long-term cell function. Inspired by photosynthetic energy generation found in nature, light-responsive artificial cells have been generated using different photosynthetic reaction complexes to functionalise membrane compartments [103-105]. Recent work by Shin and Parker used a nested vesicle containing 

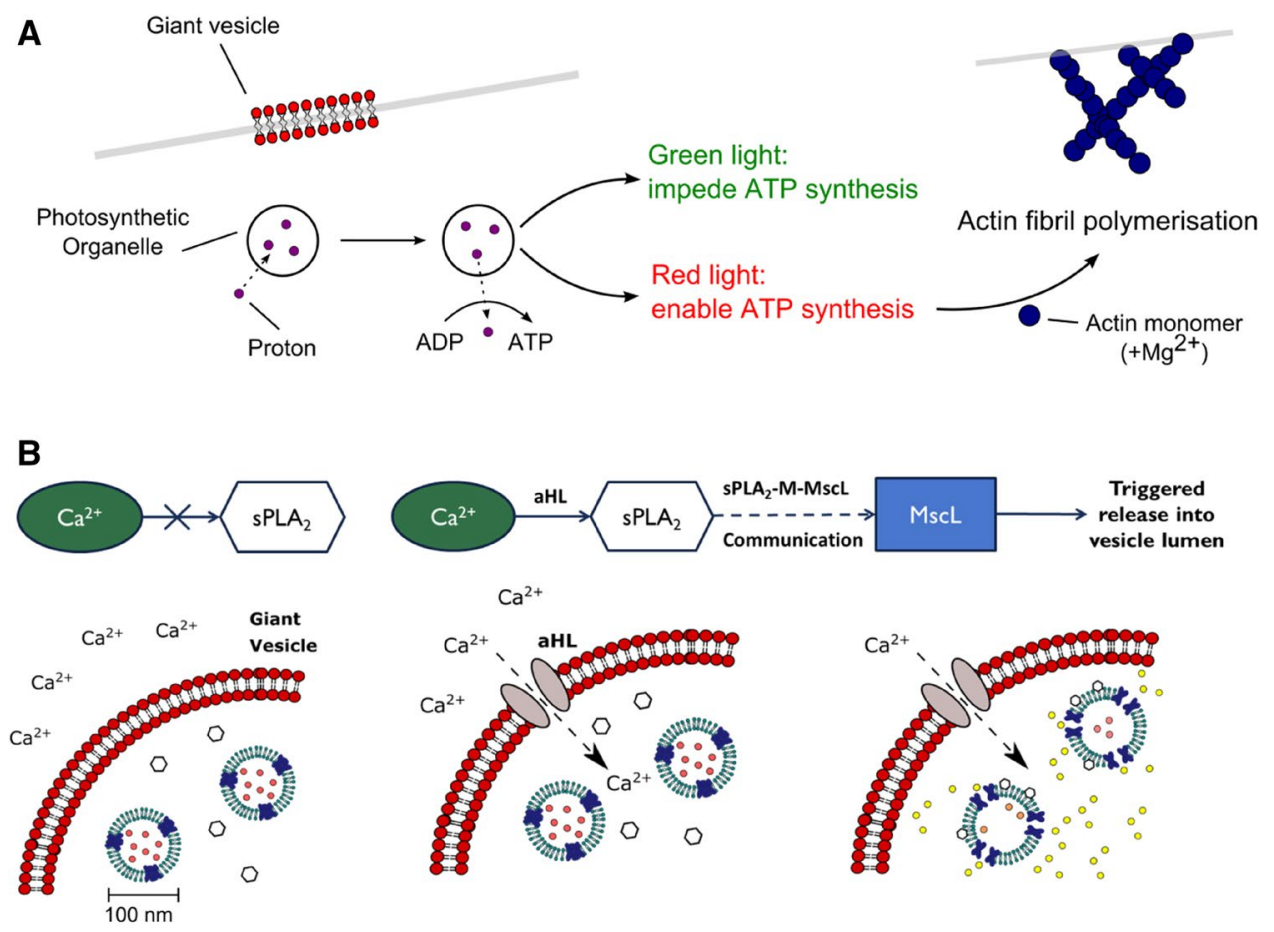

MscL Vesicles, Inactivated SPLA 2
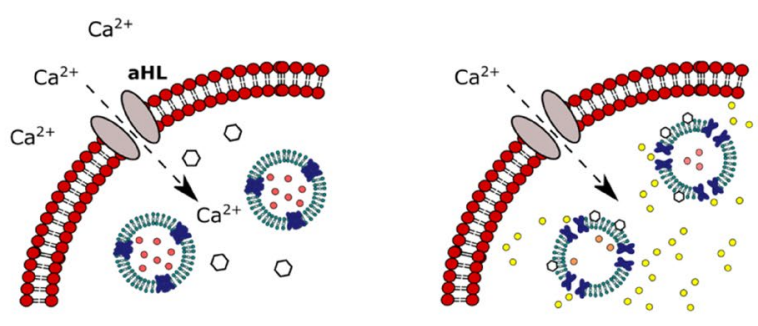

Calcium permeation activates SPLA $\quad$ SPLA2 activates MscL, releasing content

Fig. 4 Plug-and-play approaches for artificial cell design. The self-assembled nature of ACs enables a modular, or 'plug-andplay' approach to their construction. This enables the assembly of molecular networks not found in nature that can be quickly designed, tested and edited. a Engineering of a photosynthetic organelle containing proteorhodopsin (PR), photosystem II (PSII) and ATP synthase enables light-triggered actin polymerisation in a giant nested vesicle. ATP synthase generates ATP from an ADP precursor at acidic $\mathrm{pH}$. Red light facilitates this process by generating high proton concentrations through water splitting, whilst green

a photosynthetic organelle to generate ATP in response to light (Fig. 4a) [104].

Reconstituted photosystem II (PSII) was activated under red-light, generating protons in the organelle. ATP synthase then activates in response, catalysing the production of ATP in the AC. The generation of ATP in the system was further controlled by the additional reconstitution of proteorhodopsin (PR), which pumps protons out of the organelle in response to green light, inhibiting the action of ATP synthase and hence ATP concentration in the AC. Organelle-generated ATP was then used for carbon fixation, generating a biosynthetic intermediate through the ATP-ADP cycle as well as to control actin polymerisation in the vesicle. Lateral membrane organisation was additionally exploited to generate actin-mediated membrane deformation into 'teardrop' and 'mushroom'-shaped vesicles through selective actin binding to the liquid disordered lipid phase.

The photosynthetic organelle engineered in this study is a good example of one of the key strengths of light inhibits it through PR activation, leading to low proton concentrations in the organelle. Photogenerated ATP then drives actin polymerisation in the AC. $\mathbf{b}$ Construction of a synthetic signalling pathway in a nested vesicle. Calcium-dependent secretory phospholipase A2 ( $\left(\mathrm{PLA}_{2}\right)$ is inactivated through chelation in the AC. Calcium flux upon assembly of the alpha hemolysin pore then activates $\mathrm{SPLA}_{2}$, which in turn can act on the internal vesicles, generating asymmetric concentrations of lysophospholipids that drive the opening of the MscL channel, leading to content mixing in the $A C$ lumen. Figure $4 b$ reproduced from [106]

bottom-up synthetic biology over genetic engineering approaches: the ability to 'plug-and-play' with molecular components. By taking PSII from plants and PR from bacteria/archaea, two photoconverters have been combined that do not normally exist together in biology [104]. Another example of the 'plug-and-play' approach was recently developed by our laboratory, combining detergent-mediated reconstitution of membrane proteins with EPT to generate a nested (vesosome) system containing a synthetic signalling pathway (Fig. 4b) [106]. This pathway was constructed by encapsulating a previously developed interaction between secretory phospholipase A2 ( $\left(P_{2} A_{2}\right)$ and vesicles functionalised with the mechanosensitive channel of large conductance (MscL) [107] to generate a nested system. SPLA 2 activity was inhibited via calcium chelation, and the pathway was completed by functionalising the external vesicle membrane with the alpha hemolysin membrane pore. This allowed the nested vesicles to respond to an external calcium flux through sPLA ${ }_{2}$ activation and subsequent $s P L A_{2}-M-M s c L$ 
communication, leading to increased AC fluorescence upon the release of a self-quenching calcein dye through activated MscL channels. Whilst further developments to the engineered pathway are necessary to produce ACs that can respond to specific molecules in the local environment, such systems represent a new tool for controlling $A C$ processes and highlight the potential of plugand-play approaches for de novo pathway design in ACs.

\section{Future perspectives: interfacing functional membranes with biology and chemistry}

As detailed above, a wide variety of approaches exist to create functional lipid membranes that can be exploited in AC design. The biomimetic nature of such lipid systems facilitates active protein reconstitution [108] as well as a high degree of biocompatibility [109], enabling membranes to be interfaced with (and generated by) biochemical machinery such as cell-free transcription-translation systems obtained from the lysates of living cells [110]. This allows the creation of ACs with the ability to express proteins in the cell lumen [11,111-113], and has been exploited in a variety of contexts as detailed by a recent review [114].

Unlike living systems which rely on proteins for almost all functions, ACs are readily compatible with the wide range of molecules generated by synthetic chemistry and nanotechnology. These include synthetic block polymer ion channels [115] and DNA origami nanopores [116, 117] to replace the use of membrane protein pores, the creation of hybrid copolymer-lipid membranes for patterning and increased membrane stability $[118,119]$, nucleic acid cytoskeletons [120], synthetic molecules capable of signal transduction across the AC membrane without the use of protein components $[121,122]$ and nucleic acid strand displacement networks to program cell functions [123-126]. One future challenge to be considered if using synthetic molecules instead of biologically derived components in ACs is how to integrate regeneration of such components in situ without destabilising the cell itself. Solutions could be found by using green chemistry [127] as well as protein engineering approaches [128] to create new biosynthetic routes to abiotic molecules initially produced in the chemistry laboratory.

The next step in the development of ACs will require the researcher to creatively combine elements from the vast range of molecular motifs available to generate robust, switchable functions that can operate in increasingly complex environments. By employing frameworks from systems chemistry [129-131], molecular networks capable of the complex behaviour displayed by genetic circuitry operating in living systems [132] could be utilised in ACs. This would enable the creation of ACs with longer operating times capable of multiple activation cycles. In order to achieve this, developments in engineering AC architecture and replication $[133,134]$ needs to be married with significant developments in generating a protometabolism that enables cell regeneration. Just as it has evolved in living systems [135], it seems likely that membrane organization and composition in ACs will play a vital role in conducting the multiple processes necessary for life-like function.

Acknowledgements This work was supported by an Engineering and Physical Sciences Research Council (EPSRC) Centre for Doctoral Training Studentship from the Institute of Chemical Biology (Imperial College London) and an EPSRC Doctoral Prize Fellowship to J.W.H.

\section{Compliance with ethical standards}

Conflict of interest On behalf of all authors, the corresponding author states that there is no conflict of interest.

Open Access This article is licensed under a Creative Commons Attribution 4.0 International License, which permits use, sharing, adaptation, distribution and reproduction in any medium or format, as long as you give appropriate credit to the original author(s) and the source, provide a link to the Creative Commons licence, and indicate if changes were made. The images or other third party material in this article are included in the article's Creative Commons licence, unless indicated otherwise in a credit line to the material. If material is not included in the article's Creative Commons licence and your intended use is not permitted by statutory regulation or exceeds the permitted use, you will need to obtain permission directly from the copyright holder. To view a copy of this licence, visit http://creativecommons .org/licenses/by/4.0/.

\section{References}

1. Hutchison CA et al (2016) Design and synthesis of a minimal bacterial genome. Science 351:aad6253

2. Martínez-García E, de Lorenzo V (2016) The quest for the minimal bacterial genome. Curr Opin Biotechnol 42:216-224

3. Simunovic M, Evergren E, Callan-Jones A, Bassereau P (2019) Curving cells inside and out: roles of BAR domain proteins in membrane shaping and its cellular implications. Annu Rev Cell Dev Biol 35:111-129

4. Kretschmer S, Ganzinger KA, Franquelim HG, Schwille P (2019) Synthetic cell division via membrane-transforming molecular assemblies. BMC Biol 17:1-10

5. Gánti T (2003) Chemoton theory. Kluwer Academic/Plenum Publishers, Dordrecht

6. Cronin L et al (2006) The imitation game-a computational chemical approach to recognizing life. Nat Biotechnol 24:1203-1206

7. Luisi PL (1998) About various definitions of life. Origins Life Evol Biosph 28:613-622

8. Chodasewicz K (2014) Evolution, reproduction and definition of life. Theory Biosci 133:39-45

9. Turing AMI (1950) Computing machinery and intelligence. Mind LIX:433-460 
10. Lentini R et al (2017) Two-way chemical communication between artificial and natural cells. ACS Cent Sci 3:117-123

11. Noireaux V, Libchaber A (2004) A vesicle bioreactor as a step toward an artificial cell assembly. Proc Natl Acad Sci USA 101:17669-17674

12. Walde P, Cosentino K, Engel H, Stano P (2010) Giant vesicles: preparations and applications. ChemBioChem 11:848-865

13. Huang $X$ et al (2013) Interfacial assembly of protein-polymer nano-conjugates into stimulus-responsive biomimetic protocells. Nat Commun 4:2239

14. Liu X et al (2016) Hierarchical proteinosomes for programmed release of multiple components. Angew Chem Int Ed 55:7095-7100

15. Discher BM et al (1999) Polymersomes: tough vesicles made from diblock copolymers. Science 284:1143-1146

16. Peters RJRW et al (2014) Cascade reactions in multicompartmentalized polymersomes. Angew Chem Int Ed 53:146-150

17. Messager $L$ et al (2016) Biomimetic hybrid nanocontainers with selective permeability. Angew Chem Int Ed 55:11106-11109

18. Li M, Harbron RL, Weaver JVM, Binks BP, Mann S (2013) Electrostatically gated membrane permeability in inorganic protocells. Nat Chem 5:529-536

19. Fukui Y, Fujimoto K (2009) The preparation of sugar polymercoated nanocapsules by the layer-by-layer deposition on the liposome. Langmuir 25:10020-10025

20. Chandrawati R et al (2010) Engineering advanced capsosomes: maximizing the number of subcompartments, cargo retention, and temperature-triggered reaction. ACS Nano 4:1351-1361

21. Hosta-Rigau L, York-Duran M (2014) Confined multiple enzymatic (cascade) reactions within poly (dopamine)-based capsosomes. ACS Appl Mater Interfaces 6:12771-12779

22. Dora Tang T-Y et al (2014) Fatty acid membrane assembly on coacervate microdroplets as a step towards a hybrid protocell model. Nat Chem 6:527-533

23. Vieregg JR, Tang TYD (2016) Polynucleotides in cellular mimics: coacervates and lipid vesicles. Curr Opin Colloid Interface Sci 26:50-57

24. Karzbrun E, Tayar AM, Noireaux V, Bar-Ziv RH (2014) Programmable on-chip DNA compartments as artificial cells. Science 345:829-832

25. Moriizumi Y et al (2018) Hybrid cell reactor system from Escherichia coli protoplast cells and arrayed lipid bilayer chamber device. Sci Rep 8:11757

26. Izri Z, Garenne D, Noireaux V, Maeda YT (2019) Gene expression in on-chip membrane-bound artificial cells. ACS Synth Biol 8:1705-1712

27. Blain JC, Szostak JW (2014) Progress toward synthetic cells. Annu Rev Biochem 83:615-640

28. Toparlak OD, Mansy SS (2019) Progress in synthesizing protocells. Exp Biol Med 244:304-313

29. Smith R, Tanford C (1972) The critical micelle concentration of I-a-dipalmitoylphosphatidylcholine in water and water/methanol solutions. J Mol Biol 67:75-83

30. Tanford C (1978) The hydrophobic effect and the organization of living matter. Science 200:1012-1018

31. Tristram-Nagle S, Nagle JF (2004) Lipid bilayers: thermodynamics, structure, fluctuations, and interactions. Chem Phys Lipids 127:3-14

32. Allen TM, Cullis PR (2013) Liposomal drug delivery systems: from concept to clinical applications. Adv Drug Deliv Rev 65:36-48

33. Berger N, Sachse A, Bender J, Schubert R, Brandl M (2001) Filter extrusion of liposomes using different devices: comparison of liposome size, encapsulation efficiency, and process characteristics. Int J Pharm 223:55-68
34. Liang W, Levchenko TS, Torchilin VP (2004) Encapsulation of ATP into liposomes by different methods: optimization of the procedure. J Microencapsul 21:251-261

35. Schroeder A, Kost J, Barenholz Y (2009) Ultrasound, liposomes, and drug delivery: principles for using ultrasound to control the release of drugs from liposomes. Chem Phys Lipids 162:1-16

36. Angelova MI, Dimitrov DS (1986) Liposome electroformation. Faraday Discuss Chem Soc 81:303

37. Pautot S, Frisken BJ, Weitz DA (2003) Production of unilamellar vesicles using an inverted emulsion. Langmuir 19:2870-2879

38. Blosser MC, Horst BG, Keller SL (2016) CDICE method produces giant lipid vesicles under physiological conditions of charged lipids and ionic solutions. Soft Matter 12:7364-7371

39. Moga A, Yandrapalli N, Dimova R, Robinson T (2019) Optimization of inverted emulsion-base method for high-throughput production of giant unilamellar vesicles. ChemBioChem 20:1-10

40. Veatch SL, Keller SL (2002) Organization in lipid membranes containing cholesterol. Phys Rev Lett 89:268101

41. Veatch SL, Keller SL (2003) Separation of liquid phases in giant vesicles of ternary mixtures of phospholipids and cholesterol. Biophys J 85:3074-3083

42. Hamada T, Kishimoto Y, Nagasaki T, Takagi M (2011) Lateral phase separation in tense membranes. Soft Matter 7:9061

43. Guo MT, Rotem A, Heyman JA, Weitz DA (2012) Droplet microfluidics for high-throughput biological assays. Lab Chip 12:2146-2155

44. Funakoshi K, Suzuki H, Takeuchi S (2006) Lipid bilayer formation by contacting monolayers in a microfluidic device for membrane protein analysis. Anal Chem 78:8169-8174

45. Bayley $\mathrm{H}$ et al (2008) Droplet interface bilayers. Mol BioSyst 4:1191-1208

46. Miller D et al (2013) Protocell design through modular compartmentalization. J R Soc Interface 10:20130496

47. Villar G, Graham AD, Bayley H (2013) A tissue-like printed material. Science 340:48-52

48. Booth MJ, Schild VR, Graham AD, Olof SN, Bayley H (2016) Light-activated communication in synthetic tissues. Sci Adv 2:e1600056

49. Dupin A, Simmel FC (2019) Signalling and differentiation in emulsion-based multi-compartmentalized in vitro gene circuits. Nat Chem 11:32-39

50. Pautot S, Frisken BJ, Weitz DA (2003) Engineering asymmetric vesicles. Proc Natl Acad Sci USA 100:10718-10721

51. Fujii S, Matsuura T, Sunami T, Kazuta Y, Yomo T (2013) In vitro evolution of a-hemolysin using a liposome display. Proc Natl Acad Sci USA 110:16796-16801

52. Elani Y, Gee A, Law RV, Ces O (2013) Engineering multi-compartment vesicle networks. Chem Sci 4:3332

53. Thomas JM, Friddin MS, Ces O, Elani Y (2017) Programming membrane permeability using integrated membrane pores and blockers as molecular regulators. Chem Commun 53:12282-12285

54. Kubatta EA, Rehage $\mathrm{H}$ (2009) Characterization of giant vesicles formed by phase transfer processes. Colloid Polym Sci 287:1117-1122

55. Angelova M, Dimitrov DS (1988) A mechanism of liposome electroformation. Prog Colloid Polym Sci 76:59-67

56. Rabl J, Leibundgut M, Ataide SF, Haag A, Ban N (2011) Crystal structure of the eukaryotic $40 \mathrm{~S}$ ribosomal subunit in complex with initiation factor 1. Science 331:730-736

57. Silver RP, Aaronson W, Sutton A, Schneerson R (1980) Comparative analysis of plasmids and some metabolic characteristics of Escherichia coli K1 from diseased and healthy individuals. Infect Immun 29:200-206 
58. Noireaux V, Bar-Ziv R, Godefroy J, Salman H, Libchaber A (2005) Toward an artificial cell based on gene expression in vesicles. Phys Biol 2:P1-P8

59. Elani Y, Law RV, Ces O (2015) Protein synthesis in artificial cells: using compartmentalisation for spatial organisation in vesicle bioreactors. Phys Chem Chem Phys 17:15534-15537

60. Walker SA, Kennedy MT, Zasadzinski JA (1997) Encapsulation of bilayer vesicles by self-assembly. Nature 387:61-64

61. Kisak E, Coldren B, Evans C, Boyer C, Zasadzinski J (2004) The vesosome-a multicompartment drug delivery vehicle. Curr Med Chem 11:1241-1253

62. Boyer C, Zasadzinski JA (2007) Multiple lipid compartments slow vesicle contents release in lipases and serum. ACS Nano 1:176-182

63. Elani Y, Law RV, Ces O (2014) Vesicle-based artificial cells as chemical microreactors with spatially segregated reaction pathways. Nat Commun 5:5305

64. Deng NN, Yelleswarapu M, Huck WTS (2016) Monodisperse uni- and multicompartment liposomes. J Am Chem Soc 138:7584-7591

65. Villar G, Heron AJ, Bayley H (2011) Formation of droplet networks that function in aqueous environments. Nat Nanotechnol 6:803-808

66. Baxani DK et al (2016) Bilayer networks within a hydrogel shell: a robust chassis for artificial cells and a platform for membrane studies. Angew Chem Int Ed 55:14240-14245

67. Tan YC, Hettiarachchi K, Siu M, Pan YR, Lee AP (2006) Controlled microfluidic encapsulation of cells, proteins, and microbeads in lipid vesicles. J Am Chem Soc 128:5656-5658

68. Elani $Y$ et al (2018) Constructing vesicle-based artificial cells with embedded living cells as organelle-like modules. Sci Rep 8:1-8

69. Gilbert C, Ellis T (2019) Biological engineered living materials: growing functional materials with genetically programmable properties. ACS Synth Biol 58:14539-14543

70. Rodríguez-Hernández J, Lecommandoux S (2005) Reversible inside-out micellization of $\mathrm{pH}$-responsive and water-soluble vesicles based on polypeptide diblock copolymers. J Am Chem Soc 127:2026-2027

71. Mo R et al (2012) Multistage pH-responsive liposomes for mitochondrial-targeted anticancer drug delivery. Adv Mater 24:3659-3665

72. Jorgensen K, Davidsen J, Mouritsen OG (2002) Biophysical mechanisms of phospholipase A2 activation and their use in liposome-based drug delivery. FEBS Lett 531:23-27

73. Law B, Tung CH (2009) Proteolysis: a biological process adapted in drug delivery, therapy, and imaging. Bioconjug Chem 20:1683-1695

74. Zhu G, Mock JN, Aljuffali I, Cummings BS, Arnold RD (2011) Secretory phospholipase $\mathrm{A} 2$ responsive liposomes. J Pharm Sci 100:3146-3159

75. Fouladi F, Steffen KJ, Mallik S (2017) Enzyme-responsive liposomes for the delivery of anticancer drugs. Bioconjug Chem 28:857-868

76. Grüll H, Langereis S (2012) Hyperthermia-triggered drug delivery from temperature-sensitive liposomes using MRIguided high intensity focused ultrasound. J Control Release 161:317-327

77. Kim MS et al (2014) Temperature-triggered tumor-specific delivery of anticancer agents by CRGD-conjugated thermosensitive liposomes. Colloids Surf B Biointerfaces 116:17-25

78. Ong W, Yang Y, Cruciano AC, McCarley RL (2008) Redoxtriggered contents release from liposomes. J Am Chem Soc 130:14739-14744
79. Yavlovich A et al (2009) Design of liposomes containing photopolymerizable phospholipids for triggered release of contents. JTherm Anal Calorim 98:97-104

80. Lovell JF et al (2011) Porphysome nanovesicles generated by porphyrin bilayers for use as multimodal biophotonic contrast agents. Nat Mater 10:324-332

81. Schroeder A et al (2009) Ultrasound triggered release of cisplatin from liposomes in murine tumors. J Control Release 137:63-68

82. Mikhaylov G et al (2011) Ferri-liposomes as an MRI-visible drugdelivery system for targeting tumours and their microenvironment. Nat Nanotechnol 6:594-602

83. Li S, Goins B, Zhang L, Bao A (2012) Novel multifunctional theranostic liposome drug delivery system: construction, characterization, and multimodality MR, near-infrared fluorescent, and nuclear imaging. Bioconjug Chem 23:1322-1332

84. Lee Y, Thompson DH (2017) Stimuli-responsive liposomes for drug delivery. Wiley Interdiscip Rev Nanomed Nanobiotechnol 9:e1450

85. Yatvin MB, Weinstein JN, Dennis WH, Blumenthal R (1978) Design of liposomes for enhanced local release of drugs by hyperthermia. Science 202:1290-1293

86. Karamdad K et al (2018) Engineering thermoresponsive phase separated vesicles formed via emulsion phase transfer as a content-release platform. Chem Sci 9:4851-4858

87. Papahadjopoulos D, Jacobson K, Nir S, Isac I (1973) Phase transitions in phospholipid vesicles fluorescence polarization and permeability measurements concerning the effect of temperature and cholesterol. Biochim Biophys Acta Biomembr 311:330-348

88. Davidsen J, Jørgensen K, Andresen TL, Mouritsen OG (2003) Secreted phospholipase $\mathrm{A} 2$ as a new enzymatic trigger mechanism for localised liposomal drug release and absorption in diseased tissue. Biochim Biophys Acta Biomembr 1609:95-101

89. Bondurant B, Mueller A, O'Brien DF (2001) Photoinitiated destabilization of sterically stabilized liposomes. Biochim Biophys Acta Biomembr 1511:113-122

90. Bolinger PY, Stamou D, Vogel H (2004) Integrated nanoreactor systems: triggering the release and mixing of compounds inside single vesicles. J Am Chem Soc 126:8594-8595

91. Bolinger P, Stamou D, Vogel H (2008) An integrated self-assembled nanofluidic system for controlled biological chemistries. Angew Chem Int Ed 47:5544-5549

92. Hindley JW et al (2018) Light-triggered enzymatic reactions in nested vesicle reactors. Nat Commun 9:1093

93. Girard P et al (2004) A new method for the reconstitution of membrane proteins into giant unilamellar vesicles. Biophys J 87:419-429

94. Gardner PM, Winzer K, Davis BG (2009) Sugar synthesis in a protocellular model leads to a cell signalling response in bacteria. Nat Chem 1:377-383

95. Witkowska A, Jablonski L, Jahn R (2018) A convenient protocol for generating giant unilamellar vesicles containing SNARE proteins using electroformation. Sci Rep 8:9422

96. Li S, Wang X, Mu W, Han X (2019) Chemical signal communication between two protoorganelles in a lipid-based artificial cell. Anal Chem 91:6859-6864

97. Bhattacharya A, Brea RJ, Niederholtmeyer H, Devaraj NK (2019) A minimal biochemical route towards de novo formation of synthetic phospholipid membranes. Nat Commun 10:1-8

98. Gibellini F, Smith TK (2010) The Kennedy pathway-de novo synthesis of phosphatidylethanolamine and phosphatidylcholine. IUBMB Life 62:414-428

99. Rowlett VW, Margolin W (2013) The bacterial Min system. Curr Biol 23:R553-R556 
100. Loose M, Fischer-Friedrich E, Ries J, Kruse K, Schwille P (2008) Spatial regulators for bacterial cell division self-organize into surface waves in vitro. Science 320:789-792

101. Zieske K, Chwastek G, Schwille P (2016) Protein patterns and oscillations on lipid monolayers and in microdroplets. Angew Chem Int Ed 55:13455-13459

102. Litschel T, Ramm B, Maas R, Heymann M, Schwille P (2018) Beating vesicles: encapsulated protein oscillations cause dynamic membrane deformations. Angew Chem Int Ed 57:16286-16290

103. Altamura E et al (2017) Highly oriented photosynthetic reaction centers generate a proton gradient in synthetic protocells. Proc Natl Acad Sci USA 114:3837-3842

104. Lee KY et al (2018) Photosynthetic artificial organelles sustain and control ATP-dependent reactions in a protocellular system. Nat Biotechnol 36:530-535

105. Berhanu S, Ueda T, Kuruma Y (2019) Artificial photosynthetic cell producing energy for protein synthesis. Nat Commun 10:1325

106. Hindley JW et al (2019) Building a synthetic mechanosensitive signaling pathway in compartmentalized artificial cells. Proc Natl Acad Sci USA 116:16711-16716

107. Charalambous K et al (2012) Engineering de novo membranemediated protein-protein communication networks. J Am Chem Soc 134:5746-5749

108. Seddon AM, Curnow P, Booth PJ (2004) Membrane proteins, lipids and detergents: not just a soap opera. Biochim Biophys Acta Biomembr 1666:105-117

109. Bozzuto G, Molinari A (2015) Liposomes as nanomedical devices. Int J Nanomed 10:975-999

110. Zemella A, Thoring L, Hoffmeister C, Kubick S (2015) Cell-free protein synthesis: pros and cons of prokaryotic and eukaryotic systems. ChemBioChem 16:2420-2431

111. Ishikawa K, Sato K, Shima Y, Urabe I, Yomo T (2004) Expression of a cascading genetic network within liposomes. FEBS Lett 576:387-390

112. Noireaux V, Maeda YT, Libchaber A (2011) Development of an artificial cell, from self-organization to computation and selfreproduction. Proc Natl Acad Sci USA 108:3473-3480

113. Majumder $S$ et al (2017) Cell-sized mechanosensitive and biosensing compartment programmed with DNA. Chem Commun 53:7349-7352

114. Rampioni G, D’Angelo F, Leoni L, Stano P (2019) Gene-expressing liposomes as synthetic cells for molecular communication studies. Front Bioeng Biotechnol 7:1

115. Muraoka T et al (2017) Mechano-sensitive synthetic ion channels. J Am Chem Soc 139:18016-18023

116. Langecker M et al (2012) Synthetic lipid membrane channels formed by designed DNA nanostructures. Science 338:932-936

117. Burns JR, Stulz E, Howorka S (2013) Self-assembled DNA nanopores that span lipid bilayers. Nano Lett 13:2351-2356

118. Le Meins JF, Schatz C, Lecommandoux S, Sandre O (2013) Hybrid polymer/lipid vesicles: state of the art and future perspectives. Mater Today 16:397-402
119. Khan S, McCabe J, Hill K, Beales PA (2020) Biodegradable hybrid block copolymer-lipid vesicles as potential drug delivery systems. J Colloid Interface Sci 562:418-428

120. Kurokawa $C$ et al (2017) DNA cytoskeleton for stabilizing artificial cells. Proc Natl Acad Sci USA 114:7228-7233

121. Langton MJ, Keymeulen F, Ciaccia M, Williams NH, Hunter CA (2016) Controlled membrane translocation provides a mechanism for signal transduction and amplification. Nat Chem 9:426-430

122. Langton MJ, Scriven LM, Williams NH, Hunter CA (2017) Triggered release from lipid bilayer vesicles by an artificial transmembrane signal transduction system. J Am Chem Soc 139:15768-15773

123. Zhang DY, Seelig G (2011) Dynamic DNA nanotechnology using strand-displacement reactions. Nat Chem 3:103-113

124. Douglas SM, Bachelet I, Church GM (2012) A logic-gated nanorobot for targeted transport of molecular payloads. Science 335:831-834

125. Parolini L, Kotar J, Di Michele L, Mognetti BM (2016) Controlling self-assembly kinetics of DNA-functionalized liposomes using toehold exchange mechanism. ACS Nano 10:2392-2398

126. Joesaar A et al (2019) DNA-based communication in populations of synthetic protocells. Nat Nanotechnol 14:369-378

127. Anastas P, Eghbali N (2010) Green chemistry: principles and practice. Chem Soc Rev 39:301-312

128. Romero PA, Arnold FH (2009) Exploring protein fitness landscapes by directed evolution. Nat Rev Mol Cell Biol 10:866-876

129. Wehner $M$ et al (2016) An integrated design and fabrication strategy for entirely soft, autonomous robots. Nature 536:451-455

130. Pogodaev $A A$, Fernández Regueiro $C L$, Jakštaitè $M$, Hollander MJ, Huck WTS (2019) Modular design of small enzymatic reaction networks based on reversible and cleavable inhibitors. Angew Chem Int Ed 58:14539-14543

131. Maguire OR, Huck WTS (2019) On the importance of reaction networks for synthetic living systems. Emerg Topics Life Sci 3:517-527

132. Brophy JAN, Voigt CA (2014) Principles of genetic circuit design. Nat Methods 11:508-520

133. Kita $\mathrm{H}$ et al (2008) Replication of genetic information with selfencoded replicase in liposomes. ChemBioChem 9:2403-2410

134. Kurihara K et al (2011) Self-reproduction of supramolecular giant vesicles combined with the amplification of encapsulated DNA. Nat Chem 3:775-781

135. Azeloglu EU, lyengar R (2015) Signaling networks: information flow, computation, and decision making. Cold Spring Harb Perspect Biol 7:a005934

Publisher's Note Springer Nature remains neutral with regard to jurisdictional claims in published maps and institutional affiliations. 